\title{
Resultados en la cirugía por rotura del ligamento cruzado anterior en pacientes con edad pediátrica
}

\author{
DOI: http//dx.doi.org/10.37315/SOTOCAV201928054118
}

\section{SALVADOR MARÍN J*, MIRANDA GOROZARRI C**, BARCELó BAÑULS JM*, ALONSO HERNÁNDEZ J**, PALAZÓN QUEVEDO A**, MARTÍNEZ LÓPEZ JF*}

\begin{abstract}
*HOSPITAL UNIVERSITARIO SANT JOAN D'ALACANT. SERVICIO DE CIRUGÍA ORTOPÉDICA Y TRAUMATOLOGÍA. CARRETERA NACIONAL 332 S/N. 03550. SANT JOAN D'ALACANT, ALICANTE, ESPAÑA.

**HOSPITAL UNIVERSITARIO NIÑO JESÚS. SERVICIO DE CIRUGÍA ORTOPÉDICA Y TRAUMATOLOGÍA. AVENIDA MENÉNDEZ PELAYO 65. 28009. MADRID. ESPAÑA.
\end{abstract}

\section{Resumen.}

Objetivo: Describir los resultados del tratamiento y complicaciones así como establecer la relación de lesiones asociadas y el tiempo hasta la cirugía.

Métodos: Estudio retrospectivo de pacientes con fisis abiertas intervenidos mediante cirugía de reconstrucción del ligamento cruzado anterior entre Junio de 2010 y Junio de 2018.

Resultados: 39 pacientes fueron incluidos. La edad media fue de 14,7 años. El seguimiento medio fue de 42 meses. El tiempo medio hasta la cirugía fue de 10,7 meses. El aumento de este se asoció positivamente al incremento de lesiones asociadas (Test de Spearman 0,106. 1 caso sufrió un puente fisario $(2,5 \%)$ con discrepancia de longitud de miembros inferiores $(2,5 \%)$. La escala de Lysholm mostró 92 puntos de media al finalizar el seguimiento.

Conclusiones: La técnica transfisaria obtuvo buenos resultados funcionales con un bajo índice de complicaciones. El incremento del tiempo hasta la cirugía se asoció positivamente con el aumento de lesiones asociadas.

Palabras clave: Rotura de ligamento cruzado anterior, cierre fisario precoz, artroscopia de rodilla, edad pediátrica.

\section{Summary.}

Objectives: Describing the results of the trans-physeal surgical technique on anterior cruciate ligament (ACL) tear and the appearance of eventual complications and setting the index of associated lesions from the initial diagnosis to surgery diagnosis.

Methods: Retrospective study of patients under 18 intervened due to ACL injury with trans-physeal surgical technique between June 2010 and June 2018.

Results: 39 patients were included, The average age was 14.7 years (9-17). Mean follow-up was 42 months (6-104) and average time until surgery was 10,7 months (1-48). Spearman correlation test associated positively $(0.106)$ the increase in time to surgery and the increase in the number of associated injuries. There was 1 case of physeal bridge $(2.5 \%)$ with lower limbs lenght discrepancy.

Conclusion: Trans-physeal technique obtained good functional results with a low rate of complications at the end of followup in our serie. The increase in time to surgery was positively associated with the increase in associated injuries in the injured knee.

\footnotetext{
Correspondencia:

Jorge Salvador Marín Servicio de Cirugía Ortopédica y

Traumatología.

Hospital Universitario Sant Joan d'Alacant. Ctra. Nnal. 332,

Alacant-Valencia, s/n, 03550 Sant Joan d'Alacant, Alacant.

Correo electrónico: salvadormarinj@gmail.com
} 


\section{SALVADOR MARIN J y COLS. Resultados en la cirugía por rotura del ligamento cruzado anterior en pacientes con edad pediátrica}

\section{Introducción}

El inicio cada vez más temprano de la práctica deportiva en pacientes de edad pediátrica ha ocasionado un incremento de lesiones en este rango de población ${ }^{1}$. La rotura del ligamento cruzado anterior (LCA) es una de las lesiones más incapacitantes en la rodilla en el ámbito deportivo y, a pesar de no tener una alta frecuencia en pacientes menores de 18 años supone un $3,3 \%$ del total de las roturas de este ligamento ${ }^{2}$.

El tratamiento conservador de estas lesiones no ha demostrado una alta adherencia en niños. Sin embargo, si se han encontrado altas tasas de inestabilidad, así como lesiones articulares asociadas, más frecuentemente a nivel meniscal y condral ${ }^{3,4}$ por lo que suele ser de elección el tratamiento quirúrgico. En esta población debemos tener en cuenta una serie de consideraciones técnicas en cuanto a la cirugía se refiere, ya que, a diferencia del adulto, el cuidado de la fisis resulta crucial a la hora de realizar los túneles óseos.

En la bibliografía, clásicamente el manejo terapéutico en pacientes con fisis abiertas se ha basado en el índice de Tanner $^{5}$, y la edad, priorizando el uso de técnicas de preservación fisaria en estadios de Tanner 1-2 y/o edad < o igual a 12 en mujeres $\mathrm{y}<\mathrm{o}$ igual a 11 en varones $\mathrm{y}$ dejando las técnicas de reconstrucción transfisaria sin pastilla ósea a partir del estadios de Tanner 3-4 y/o edad a partir de 13 en mujeres y 12 años en varones.

El objetivo principal de nuestro estudio es describir los resultados del tratamiento quirúrgico en nuestra serie y la aparición de eventuales complicaciones a nivel fisario. El objetivo secundario es establecer el índice de lesiones asociadas que aparecieron entre el momento del diagnóstico por resonancia magnética nuclear (RMN) y la cirugía.

\section{Material y Método}

Estudio analítico retrospectivo de una serie de casos intervenidos en un Hospital Infantil Universitario entre Junio de 2010 y Junio de 2018.

Los criterios de inclusión fueron pacientes menores de 18 años, con fisis abiertas en el momento del diagnóstico y que fueron intervenidos mediante cirugía de reconstrucción del ligamento cruzado anterior con técnica transfisaria. Se excluyeron los pacientes con un seguimiento inferior a 6 meses y otras técnicas así como cirugías de revisión.

Se analizaron las variables edad, sexo, lateralidad, mecanismo de lesión, diagnóstico en RMN, tiempo hasta la cirugía, diagnóstico intraoperatorio con artroscopia, lesiones asociadas, complicaciones, presencia de discrepancia de longitud de miembros inferiores, eje de carga o cierre fisario precoz, re-intervención, escala de Lysholm, rigidez residual y sensación de inestabilidad al finalizar el seguimiento. Se analizó la relación entre el tiempo hasta la cirugía y la aparición de lesiones asociadas (dividas por número/unidad en meniscales, condrales o ligamentosas) utilizando la prueba $T$ de muestras emparejadas, el test de correlación de Spearman y el estimador de supervivencia de Kaplan-Meyer. Las variables cuantitativas se presentan con medias, rango y desviación estándar, y las cualitativas con porcentajes.

La técnica quirúrgica utilizada para el tratamiento de la rotura del LCA fue la reconstrucción del ligamento cruzado anterior con plastia autóloga de isquiotibiales utilizando el sistema transfisario monotunel desde la tibia. El protocolo de rehabilitación fue el mismo en todos los pacientes: Se utilizó una ortesis articulada con movilidad restringida $0^{\circ}$ $30^{\circ}$ y descarga durante 2 semanas. Tras retirar la ortesis se incrementó la movilidad progresviamente y se inició la carga parcial con muletas abandonándolas paulatinamente.

El análisis estadístico se realiza con el programa SPSS versión 18.0 (SPSS Inc., Chicago, USA).

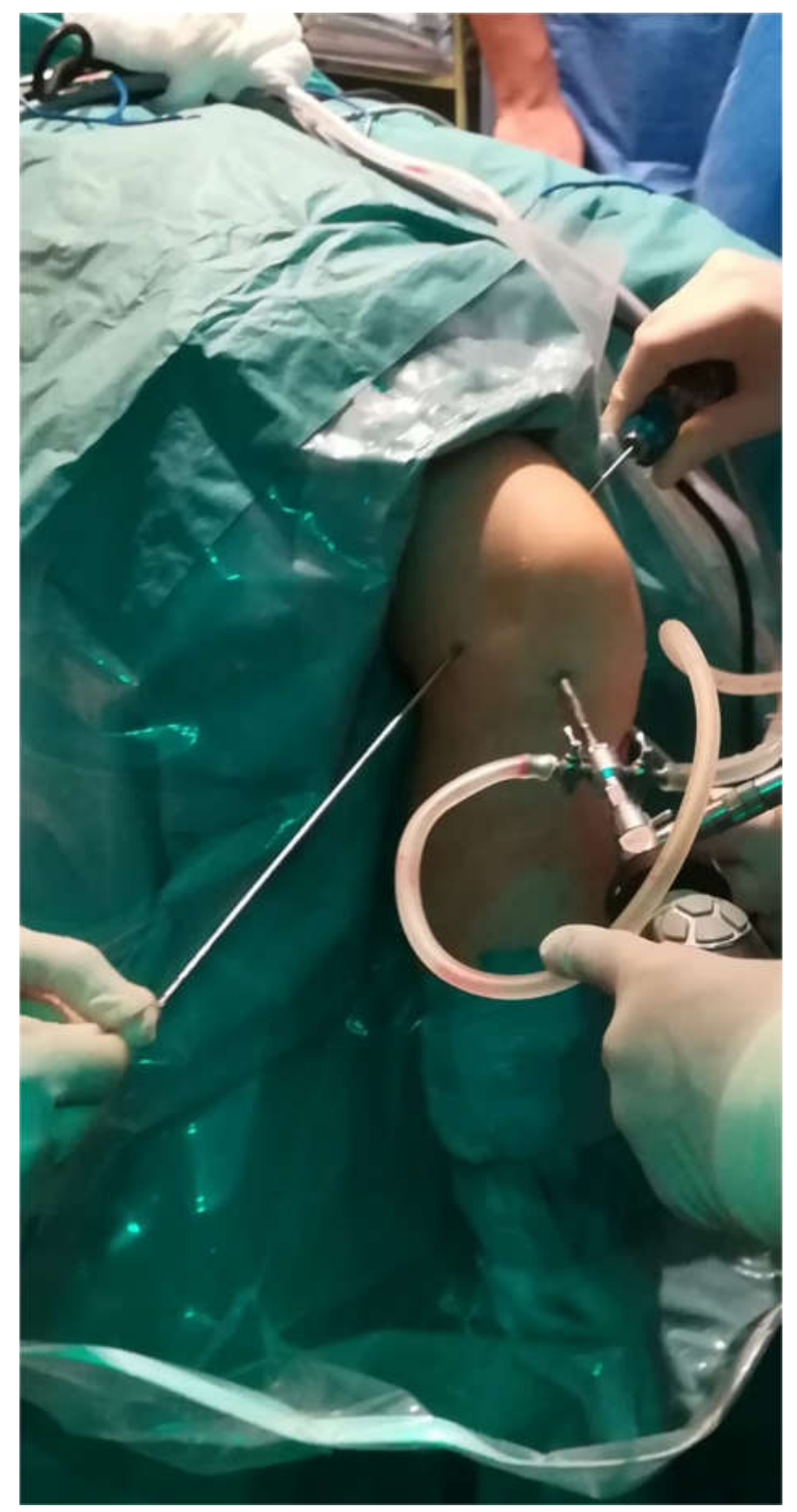

Figura 1. Imagen durante la cirugía. 


\section{Resultados}

Han sido incluidos 39 pacientes en el estudio, 16 de ellos eran niñas (41\%) y 23 eran niños (59\%). La edad media fue de 14,7 años (9-17). El mecanismo de lesión más frecuente fue el accidente deportivo en contexto de la práctica del fútbol (n:14), seguido de la práctica del baloncesto $(\mathrm{n}: 8)$.

El periodo de seguimiento medio fue de 42 meses. El tiempo medio hasta la cirugía fue de 10,7 meses. La curva de supervivencia de Kaplan-Meyer mostró que en el $41 \%(n: 16)$ de los casos se diagnosticó con artroscopia al menos una lesión asociada que no se apreció en diagnóstico inicial con RMN. El test de correlación de Spearman asoció positivamente $(0,106)$ el incremento del tiempo hasta la cirugía y el aumento del número de lesiones asociadas $(p=0,53)$. El test de muestras emparejadas mostró un incremento medio de 0,7 lesiones en el momento de la cirugía $(p<0.005)$.

Al finalizar el seguimiento, la escala Lysholm media fue de 92 puntos (39-100). El 10,25\% de los pacientes (n:4) no lograron la extensión completa de la rodilla intervenida y el $30 \%$ (n:12) no lograron la flexión completa comparando ambos parámetros con la rodilla contralateral. 8 pacientes $(20,5 \%)$ tenían sensación de inestabilidad y el $100 \%$ de los pacientes retomaron algún tipo de actividad deportiva. El $75 \%$ de los pacientes lograron volver a realizar actividades deportivas de impacto.

Se dio un $12,8 \%$ de complicaciones menores durante el seguimiento. El $10,5 \%$ de los pacientes (4) sufrieron una nueva rotura de la plastia durante el periodo de seguimiento, y en 5 pacientes su realizó una segunda cirugía a causa de complicaciones o nuevas lesiones que surgieron durante el seguimiento. Al finalizar el seguimiento, atendiendo a los resultados radiográficos se apreció 1 caso de puente fisario en la fisis tibial que evolucionó con discrepancia de longitud de miembros inferiores asociada (2,5\%). No se apreciaron alteraciones del eje del miembro inferior durante el seguimiento.

\section{Discusión}

El cuidado de la fisis de crecimiento en pacientes con edad pediátrica en la realización de los túneles óseos es prioritario cuando se realiza la cirugía de reconstrucción del ligamento cruzado anterior de la rodilla. Las fisis de la rodilla aportan el $65 \%$ del total del crecimiento de los miembros inferiores. En la bibliografía, autores como Janary y Seil ${ }^{6}$ han mostrado que el riesgo de alteraciones en el crecimiento óseo se incrementa con diversos factores. Uno de ellos se da en el caso en el que el túnel tibial ocupe más del $7 \%$ de la superficie fisaria de la tibia. La existencia de una pastilla ósea ocupando la fisis es otro factor que aumenta el riesgo de cierre fisario precoz. Por otro lado, una tensión excesiva del injerto y un túnel no centrado (periférico) y oblicuo han sido descritos como otros 2 factores que favorecen la aparición de alteraciones en el crecimiento fisario ${ }^{6,7}$. Kercher et al ${ }^{8}$ por otro lado demostraron que túneles de $8 \mathrm{~mm}$ de diámetro a través de la fisis afectan al $3 \%$ de la superficie fisaria en el caso de la tibia, lo que está por debajo del $7 \%$ previamente mencionado.

La tasa de discrepancia de longitud en miembros inferiores en la ligamentoplastia del LCA está en torno al $2 \%$ según el estudio de Tovar-Cuellar et al ${ }^{9}$. Además han mostrado que desviaciones axiales del eje se dan en el $2 \%$ de los casos y el índice de rotura de la plastia se sitúa en torno al $4-10 \%$ en este tipo de cirugía. Estos resultados no distan de los obtenidos en nuestro estudio, en los que hemos encontrado 1 único caso de puente fisario con discrepancia de longitud de miembros inferiores asociada, que tuvo que ser intervenido mediante epifisiodesis, lo que supone una afectación de'2,5\% de los pacientes de nuestro estudio, y un $10,5 \%$ del total sufrieron una re-rotura de la plastia a lo largo del seguimiento medio de 42 meses.

En la actualidad, diversos autores están realizando técnicas de reconstrucción del LCA en pacientes con fisis abiertas con túneles puramente epifisarios 0 totalmente a través de la superficie fisaria; técnicas mixtas (túnel puramente epifisario en tibia y túnel transfisario en fémur) o viceversa ${ }^{10}$. Las 3 técnicas han obtenido buenos resultados en la bibliografía aunque no existen actualmente estudios prospectivos comparativos ni resultados a largo plazo en muchos análisis. Se han apreciado bajas tasas de lesión fisaria con tasas de ruptura de la plastia aunque similares a la técnica transfisaria $(10 \%)$ realizada en nuestro estudio.

A la vista de los resultados de nuestro trabajo, y acorde con la bibliografía, con un correcto cuidado de la fisis y realizando los túneles en la posición óptima, las complicaciones fisarias a largo plazo son escasas y no distan de las técnicas epifisarias o mixtas actualmente, más complejas técnicamente y no exentas de riesgos y complicaciones. A la hora de la elección del tratamiento la madurez ósea es clave, reservando estas técnicas para pacientes en edades muy tempranas con elevado potencial de crecimiento, ya que, teóricamente, el riesgo de daño a la fisis es menor, aunque actualmente la falta de evidencia en estudios en cuanto a la aparición de complicaciones indica que no hay ventajas en su uso.

\section{Conclusión}

La técnica transfisaria obtuvo buenos resultados funcionales con un bajo índice de complicaciones al finalizar el seguimiento en nuestra serie. El incremento del tiempo hasta la cirugía se asoció positivamente con el incremento de lesiones asociadas en la rodilla afecta. 
SALVADOR MARIN J y COLS. Resultados en la cirugía por rotura del ligamento cruzado anterior en pacientes con edad pediátrica

\section{Bibliografía}

1. Frank JS, Gambacorta PL. Anterior cruciate ligament injuries in the skeletally immature athlete: diagnosis and management. J Am Acad Orthop Surg 2013; 21(2):78-87.

2. Arendt EA, Dick R. Knee injury patterns among men and women in collegiate basketball and soccer: NCAA data and review of literature. Am J Sports Med 1995; 23:694-701.

3. Vavken P, Tepolt FA, Kocher MS. Concurrent Meniscal and Chondral Injuries in Pediatric and Adolescent Patients Undergoing ACL Reconstruction. J Pediatr Orthop 2018 Feb; 38(2):105-9.

4. Beasley LS, Chudik SC. Anterior cruciate ligament injury in children: update of current treatment options. Curr Opin Pediatr 2003 Feb; 15(1):45-52.

5. Fabricant PD, Kocher MS. Management of ACL Injuries in Children and Adolescents. J Bone Joint Surg Am 2017; 99(7):600-12.

6. Janarv PM, Wikström B, Hirsch G. The influence of transphyseal drilling and tendon grafting on bone growth: an experimental study in the rabbit. J Pediatr Orthop 1998; 18(2):149-54.

7. Seil R, Weitz FK, Pape D. Surgical-experimental principles of anterior cruciate ligament (ACL) reconstruction with open growth plates. J Exp Orthop 2015; 2(1):11.

8. Kercher J, Xerogeanes J, Tannenbaum A, Al-Hakim R, Black JC, Zhao J. Anterior cruciate ligament reconstruction in the skeletally immature: an anatomical study utilizing 3-dimensional magnetic resonance imaging reconstructions. J Pediatr Orthop 2009; 29(2):124-9.

9. Tovar-Cuellar W, Galván-Villamarín F, Ortiz-Morales J. Complications associated with the techniques for anterior cruciate ligament reconstruction in patients under 18 years old: a systematic review. Rev Esp Cir Ortop Traumatol 2018; 62(1):55-64.

10. Dekker TJ, Rush JK, Schmitz MR. What's New in Pediatric and Adolescent Anterior Cruciate Ligament Injuries? J Pediatr Orthop 2018; 38(3):185-92. 